\title{
Direct Numerical Simulation of Magnetic Particles Suspended in a Newtonian Fluid Exhibiting Finite Inertia Under SAOS
}

\author{
M.R. Hashemi ${ }^{\mathrm{a}}$, M.T. Manzari ${ }^{\mathrm{a}, *}$, R. Fatehi ${ }^{\mathrm{b}}$ \\ ${ }^{a}$ Center of Excellence in Energy Conversion, School of Mechanical Engineering, Sharif University of Technology, \\ Tehran, Iran. \\ ${ }^{b}$ Department of Mechanical Engineering, Persian Gulf University, Bushehr 75168, Iran.
}

\begin{abstract}
A direct numerical simulation approach is utilized to understand the oscillatory shear rheology of a confined suspension of magnetic chains formed by paramagnetic circular cylinders under the influence of an external magnetic field. The common assumption of gap-spanning chains made in the literature is relaxed in this work, so that a fully suspended (periodic) array of magnetic chains is formed. In this sense, the effective rheological parameters are only influenced through a layer of fluid adjacent to the walls. All tests are conducted at very low but finite particle Reynolds numbers, and typical inertial effects are discussed. The main aim of the present study is to investigate the apparent viscoelasticity of the system as a function of the external magnetic field and frequency of the input strain. This work concentrates on cases with large blockage ratio in order to have pronounced viscoelastic behaviours.
\end{abstract}

Keywords:

Magnetorheology, Small amplitude oscillatory shear, Magnetic chains suspension

\section{Introduction}

A magnetorheological (MR) fluid is a suspension of micron-sized magnetizable solid particles in a non-magnetic fluid, usually used in dampers and actuators with a fast response to a feedback

*Corresponding author. Tel: +98 216616 5689; Fax: +98 2166000021
Email address: mtmanzari@sharif.edu (M.T. Manzari)

Preprint submitted to Journal of Non-Newtonian Fluid Mechanics

January 17, 2018 
signal $[1,2]$. In recent years, MR fluids have received considerable attention due to their controllable rheology by adjusting the external magnetic field $[3,4]$. At the onset of applying an external magnetic field, suspended solid particles are actuated to be arranged in chain-like structures [5] which resist flow of the suspending fluid, and typically result in a solid-like behaviour [6]. By increasing the driving force, solid-like structures [7] are stretched more and eventually break up at a certain yield stress [8]. It is clear that the evolution of micro-structures significantly affects the rheological behaviour of such systems.

In practice, the Bingham plastic model is usually used to represent the rheology of a commercial MR fluid [1]. In the pre-yield state, a solid-like behaviour is expected with a shear modulus proportional to the magnetic field intensity [9]. The yield stress is determined as a function of the Mason number defined as the ratio of hydrodynamic to magnetic forces [10]. The concentration [11] and dispersion in the size [12] of solid particles can also affect the yield stress. Multiple yield stresses [11] and a thixotropic rheology [13] are also possible due to the progressive changes in the chain-like structures. In the post-yield state, a MR fluid is generally modeled as a purely viscous Newtonian medium [14]. However, for a more accurate result, the Herschel-Bulkley model can be used for MR fluids based on observation of shear thinning/thickening behaviour in the post-yield state [15].

Nevertheless, MR fluids exhibit a viscoelastic behaviour; the storage modulus is associated with the magnetostatic energy stored in chain-like structures, while the loss modulus is related to the viscous dissipation [16]. These moduli are functions of the frequency of the exerted strain [17] and increase by increasing the magnetic field intensity [18]. This has been thoroughly investigated using oscillatory shear rheometry. There is a rich literature addressing the viscoelasticity of MR fluids in the pre-yield state $[19,20]$. In practice, the most reliable means to determine the rheological behavior of a MR fluid is to conduct an experimental rheometry.

Beyond experimental analyses, numerical simulation [21] is a feasible means to study the rheological behaviour of a MR fluids as a function of its micro-structure. The very first assumption 
commonly made in the modeling of MR fluids is that magnetic chains span the whole width of the test domain. In this sense, deformation of the chains would be affine $[22,23,24]$ and the ending solid particles are the means of exerting force on the walls of a test domain [25]. Therefore, the stress response is a measure of the magnetic bonds in the chain-like structures [3] while the hydrodynamic interactions are negligible [21]. However, this assumption is justifiable only for the pre-yield state.

In the post-yield state, the large solid structures break down $[26,27]$ into smaller magnetic chains that do not necessarily span the whole width of the flow domain $[28,29,30]$. These chains affect the rheology of MR fluids greatly by interacting with the suspending fluid flow which determines the hydrodynamic stresses exerted on the walls [31]. Generally, the size and concentration of solid particles as well as the intensity of magnetic field and hydrodynamic forces determine the length of structures formed in a MR fluid [32]. Under the influence of a rather low strength magnetic field, there is a better chance for "non-gap-spanning" chain-like structures, which are detached from walls, to be formed [33]. In the literature [11], it is discussed that carbonyl iron particles (with a relative permeability of about $10^{3}$ ) are expected to form non-gap-spanning magnetic clusters when intensity of the external magnetic field is significantly lower than $10^{4} \mathrm{~A} / \mathrm{m}$. The number of such structures also increases by decreasing the concentration of suspended particles [16]. Despite the essential role of non-gap-spanning chains in determining the post-yield rheology of MR fluids, they are scarcely addressed in the literature. Numerical simulation is a convenient tool for this purpose.

Different variants of the conventional particle dynamics method have been used for numerical simulation of MR fluids $[34,35,36]$ especially in the pre-yield state. To reliably simulate the suspension of non-gap-spanning magnetic chains where the coupled hydrodynamic and magnetic interactions play an essential role, a direct numerical simulation (DNS) should be utilized. However, DNS is associated with a high and sometimes a prohibitive computational cost. Therefore, in order to achieve a desirable accuracy with practical computational costs, proper assumptions are needed to simplify the physical model. In 2012, Kang et al. [31] analyzed the apparent steady shear viscosity of a periodic array of non-gap-spanning magnetic chains using a DNS approach. They 
found that by decreasing the Mason number, the apparent viscosity increases up to a maximum value corresponding to a plateau in viscosity-Mason curves. This value is correlated to the blockage ratio of the magnetic chains suspended in the channel and volume fraction of the solid particles [37]. Later in 2015, Hashemi et al. [37] reported the viscoelastic response of a similar system under an oscillatory shear flow.

Using a DNS approach, it is also possible to include the inertia of both the suspending fluid and the suspended solid particles. Inertia has a negligible role in a steady shear, however, inertial effects are intensified by increasing frequency and/or amplitude of the input strain (rate), and/or the height of the test domain in an oscillatory shear rheometry [38, 39]. Therefore, one cannot completely prevent inertia from affecting the measured viscoelastic properties of fluids [40]. Inertia leads to a non-uniform velocity gradient and consequently alters the stress response [41] which was revealed to further reduce the apparent elasticity of a viscoelastic fluid [42]. For MR fluids in the pre-yield state, inertial effects are negligible due to the imposed restriction on the movement of solid particles, however, inertia can play a significant role in the post-yield state.

In the present work, the apparent rheological behaviour of a confined array of non-gap-spanning magnetic chain is investigated using a DNS approach. Circular paramagnetic solid particles are suspended in a two-dimensional periodic channel and the apparent stress response is computed as an average of the hydrodynamic stress exerted on the confining walls. A similar configuration was first used in [31]. Previous studies [31, 37] are extended by further investigating the apparent viscoelasticity of the system influenced by a constant external magnetic field and inertia under an oscillatory shear test. Amplitude of the input strain is small enough to avoid rupture and reformation of the magnetic chains, and keeping the stress response in the linear regime. Considering an initially symmetric configuration and the flow direction which is perpendicular to the external magnetic field, solid particles retain their chain-like structure. A rather large blockage ratio is chosen so that clearly distinctive results can be obtained for different combinations of the input parameters. In all cases, inertia plays a crucial role and is characterized by the Reynolds number 
since all solid particles are assumed to be neutrally buoyant [43]. It must be noted that although a more realistic three-dimensional model of spherical magnetic particles might lead to quantitatively different results, the present two-dimensional model is expected to exhibit similar physical features.

In the following sections, first, a brief description of the physical modeling of the problem is presented. The theoretical background of the oscillatory shear rheometry and the effects of inertia on the apparent stress response are explained next. The apparent viscoelasticity of a suspension of magnetically attracted solid bodies under an oscillatory shear are discussed afterwards. The apparent viscoelastic moduli are studied as functions of the intensity of magnetic field and the frequency of the input strain.

\section{Physical Modeling}

The ultimate goal of this study is to understand the rheological behaviour of fully suspended (para-)magnetic solid particles forming chain-like structures. This aim is achieved using a direct numerical simulation (DNS) approach and employing the least necessary assumptions in the modeling of the problem. Computations are performed for a two-dimensional domain. In the following, the simplified model is described and the numerical method is briefly presented.

\subsection{Non-gap-spanning Magnetic Chains}

Here, a series of paramagnetic solid bodies are suspended in a channel filled with a Newtonian fluid and a small amplitude oscillatory shear (SAOS) test is conducted. To establish a correlation between the measured rheological parameters and the assumed micro-structure, it is necessary to avoid structural reconfiguration of the magnetic chain during simulations.

It is known that the chaining mechanism of a series of suspended magnetic solid particles depends intrinsically on the configuration of these particles [44]. Having known this, a configuration is chosen which is symmetric in the flow direction; thus, the rejoining of the adjacent chains is prevented. Also, the amplitude and frequency of the exerted strain are kept small so that no rupture occurs 


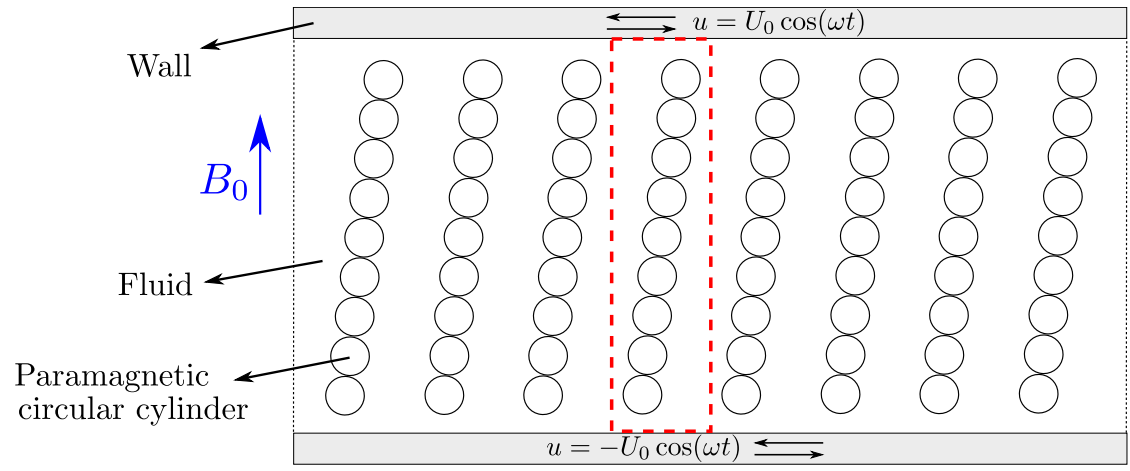

(a)

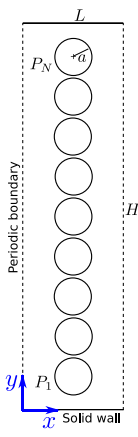

(b)

Figure 1: Schematic of (a) the suspension of magnetic solid particles shearing in a channel with oscillating solid walls and (b) the initial configuration of the particles in the computational domain. The computational domain is marked by dashed-lines in (a).

and consequently a chain-like structure is retained. A schematic of the simplified model is shown in Fig. 1a. Figure 1b illustrates the computational domain and the initial arrangement of the solid particles.

In this work, $N$ paramagnetic neutrally buoyant circular cylinders are suspended in a periodic channel filled with a Newtonian suspending fluid and an oscillatory shear is imposed to the system by controlling velocity, $u$, of the channel walls. A uniform vertically aligned external magnetic field with intensity of $H_{0}$ actuates the paramagnetic solids to be arranged in chains. The apparent rheological behaviour of the system is studied by measuring the average shear stress exerted on the (bottom) wall as

$$
\bar{\sigma}_{x y}=\frac{1}{L} \int_{y=0} \sigma_{x y}(x) d x,
$$

where $\sigma_{x y}$ is the local value of the shear stress. A similar system was considered by Kang et al. [31] in order to investigate the response of a magnetic suspension subject to a steady shear. It must be noted that in the following, the over-bar sign is omitted for brevity. 


\subsection{Governing Equations}

In the present DNS approach, fluid flow is simulated using a weakly compressible Smoothed Particle Hydrodynamics (SPH) method [45]. In SPH [46], the governing equations are written in the Lagrangian framework. The conservation of momentum reads

$$
\rho \frac{d \mathbf{v}}{d t}=-\nabla p+\eta_{0} \nabla^{2} \mathbf{v}
$$

and the continuity becomes

$$
\frac{d \rho}{d t}=-\rho \nabla \cdot \mathbf{v}
$$

Here, $\mathbf{v}$ is the velocity vector, and $p$ is pressure. No-slip boundary condition is imposed on all solid surfaces and all test-cases are solved in two-dimensional domains. In a suspension of magnetic solid particles, the time-scale associated with the movement of particles is much larger than the

electromagnetic time-scale. In this sense, the time-dependency of the electromagnetic field can be neglected [34] and the magnetostatic Maxwell's equations govern the magnetic field [47] as

$$
\nabla \times \mathbf{H}=0
$$

for the magnetic field intensity, $\mathbf{H}$, and

$$
\nabla \cdot \mathbf{B}=0
$$

for the magnetic flux density, B.

Here, solid particles are considered to be made of a paramagnetic substance, for which a linear constitutive equation can be written as [48]

$$
\mathbf{B}=\mu \mathbf{H} .
$$

The magnetic permeability, $\mu$, is considered to be constant for an isotropic paramagnetic material far below the magnetic saturation limit [49]. Considering $\mu_{0}$ to be the magnetic permeability of the suspending fluid, the relative permeability and the magnetic susceptibility of the solid particles are $\beta=\mu_{s} / \mu_{0}$ and $\chi=\beta-1$, respectively. 


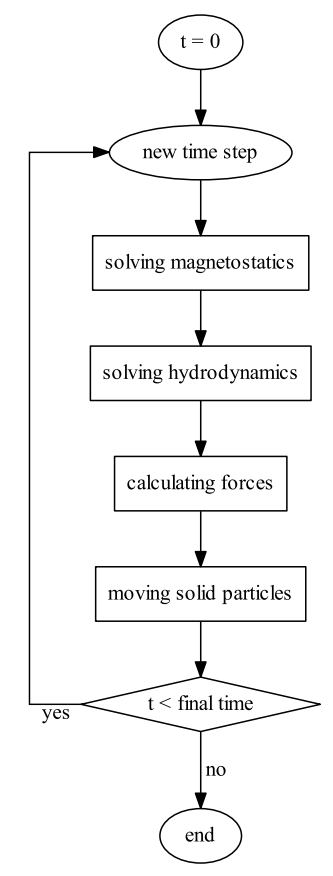

Figure 2: A flowchart of the numerical algorithm.

The system of equations (4) and (5) satisfies the conservation of the magnetic flux across any surface and the continuity of the magnetic field along any interface [47]. In the absence of free current, Maxwell's equations are combined utilizing a scalar potential (in two-dimensions) $\phi$ as

$$
\nabla \cdot(\mu \nabla \phi)=0
$$

where $\mathbf{H}=\nabla \phi$. Equation (7) along with the corresponding boundary conditions $[34,37]$ are discretized on the computational domain using the SPH method and solved for $\phi$. The magnetic forces are then calculated by integrating the Maxwell stress tensor along every fluid-solid interface as described in [37].

A fully explicit numerical algorithm is used in the context of SPH to solve the governing equations coupled with the motion of solid particles as presented in Fig. 2. As a brief description of the numerical algorithm, it can be noted that in every time-step, the governing equations are solved, the magnetic and hydrodynamic forces are calculated, and the solid particles are moved accord- 
ingly. Once the new configuration is obtained, the procedure is repeated for a new time-step. The numerical method was thoroughly described in [37].

\subsection{Non-dimensional Parameters}

For a MR fluid, the behaviour of the system is characterized by two non-dimensional parameters; the Mason number which is a measure of viscous forces versus the magnetic field strength [50], and the Reynolds number which is a measure of inertia. For a steady shear flow, the Mason number is defined as [51]

$$
M n=\frac{\dot{\gamma}_{0} \eta_{0}}{\mu_{0} \hat{\beta}^{2} H_{0}^{2}},
$$

where $\hat{\beta}=\chi /(3+\chi)$ is the effective polarization. In this way, $M n \rightarrow \infty$ corresponds to a nonmagnetic system and $M n \rightarrow 0$ refers to a non-viscous magnetic system. The smaller the Mason number, the stronger the magnetic field and vice versa. For an oscillatory shear problem, a modified Mason number can also be defined based on frequency as

$$
M n^{*}=\frac{\omega \eta_{0}}{\mu_{0} \hat{\beta}^{2} H_{0}^{2}}
$$

In the following, $M n^{*}$ is used as a measure of the strength of the external magnetic field when $\dot{\gamma}_{0}$ is variable.

The Reynolds number is defined at both the particle scale, $R e_{p}=\rho \dot{\gamma}_{0} a^{2} / \eta_{0}$, and the channel scale, $R e=\rho \dot{\gamma}_{0} H^{2} / \eta_{0}$, where $\rho$ is fluid density, $\dot{\gamma}_{0}$ denotes the amplitude of the strain-rate, and $\eta_{0}$ is dynamic viscosity of the suspending fluid. Since solid particles are neutrally buoyant, the particle Reynolds number, $R e_{p}$, characterizes the inertial movement of particles, while $R e$ characterizes the bulk flow of the system in the presence of inertia.

\section{Oscillatory Shear Rheometry}

As discussed before, a suspension of magnetic solid particles under the influence of an external magnetic field behaves as a viscoelastic material. In this sense, dynamic oscillatory shear tests are conducted to measure the rheological behaviour of the system. When subjected to an oscillatory 
shear strain, $\gamma(t)=\gamma_{0} \sin (\omega t)$, the stress response of a viscoelastic material in the linear region (for a rather small strain amplitude) can be generally described as [52]

$$
\sigma_{x y}=\left|G^{*}\right| \gamma_{0} \sin (\omega t+\Psi)
$$

The right-hand side of Eq. (10) can be rewritten as sum of two terms; one in-phase with the input strain, $\sigma_{x y}^{\prime}=\left|G^{*}\right| \gamma_{0} \cos (\Psi) \sin (\omega t)$, and the other in-phase with the strain rate, $\sigma_{x y}^{\prime \prime}=$ $\left|G^{*}\right| \gamma_{0} \sin (\Psi) \cos (\omega t)$. It is simple to show that in a complete shear cycle, the odd part of stress, $\sigma_{x y}^{\prime}$, and the even part, $\sigma_{x y}^{\prime \prime}$ make contributions to the storage and loss of energy, respectively. Consequently, the storage and loss moduli are respectively defined as $G^{\prime}=\left|G^{*}\right| \cos (\Psi)$ and $G^{\prime \prime}=$ $\left|G^{*}\right| \sin (\Psi)$. Rewriting the equations for the rate of shear strain, in the same manner, dynamic viscosities are defined as $\eta^{\prime \prime}=G^{\prime} / \omega$ and $\eta^{\prime}=G^{\prime \prime} / \omega$.

In rheological measurements, there are always concerns about misleading observations sourced from inertia [42]. At a finite Reynolds number for a rather large channel width, there is a nonuniform time-dependent velocity distribution across the channel. The first consequence can be an increased viscous dissipation [53] or equivalently a larger $G^{\prime \prime}$ compared to a uniformly sheared bulk of fluid. Nevertheless, the presence of inertia may lead to a decrease in the amplitude of the stress response for a polymeric melt [38].

At a vanishing Reynolds number, for a viscoelastic material subject to an oscillatory shear, a phase angle of $0 \leq \Psi \leq \pi / 2$ is expected. When inertia is dominant, a phase angle larger than $\pi / 2$ also emerges which is misleading and gives an apparently negative value for $G^{\prime}$. Schematic Lissajous-Bowditch curves point out the issue in Fig. 3.

In order to provide an instance of the issue, a test-case is solved for a pure Newtonian fluid at different Reynolds numbers. A bulk of Newtonian fluid with density $\rho_{0}$ and viscosity $\eta_{0}$ is sheared in an infinitely long channel with height $H=0.025(m)$ and walls that oscillate in opposite directions with $u= \pm U_{0} \cos (\omega t)$. The geometry as schematically shown in Fig. 4 , is the same as the main test-cases considered in this paper, however, here without solid particles. The Reynolds number, 


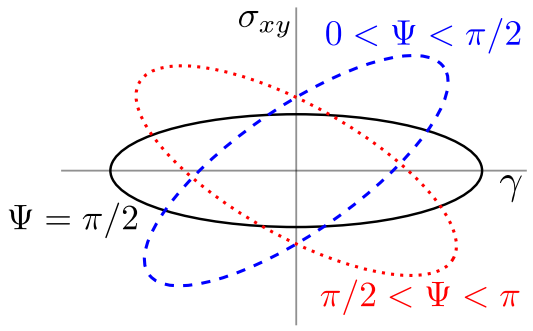

Figure 3: Schematic of the stress-strain Lissajous-Bowditch curves for different ranges of $\Psi$.

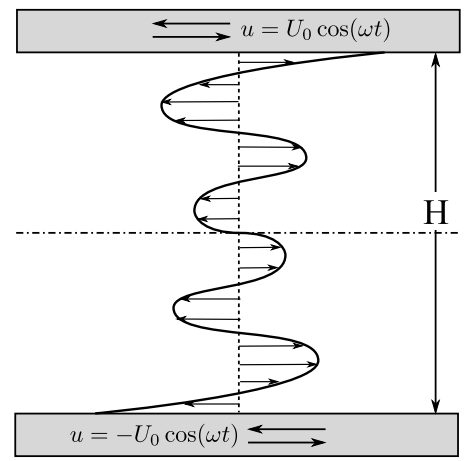

Figure 4: Schematic of a pure Newtonian fluid shearing in a channel with oscillating boundaries. 


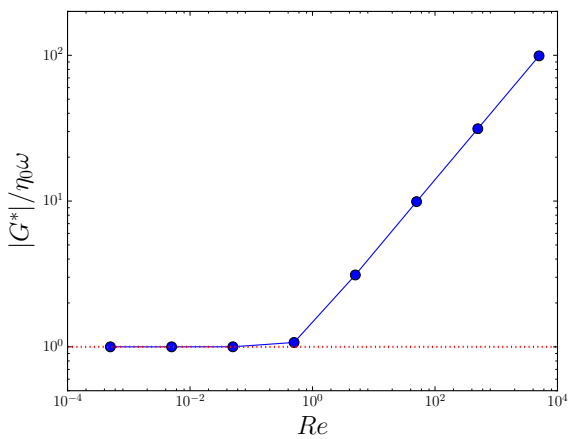

(a)

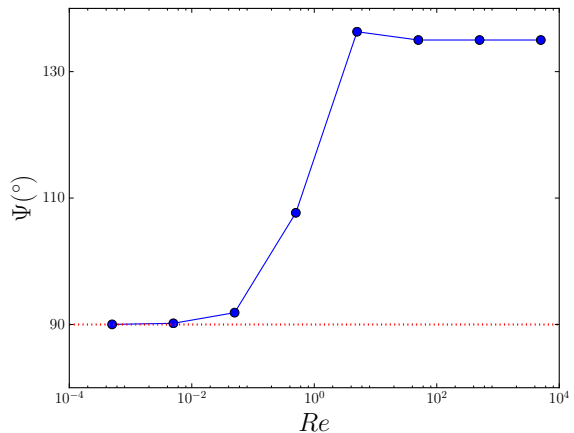

(b)

Figure 5: (a) Normalized $\left|G^{*}\right|$ and (b) phase angle obtained for a pure Newtonian fluid under oscillatory shear.

$R e$, is changed by altering $\rho_{0}$ and keeping all other variables constant. Here, the amplitude of the input strain-rate and the oscillating frequency are $\dot{\gamma}_{0}=2 U_{0} / H=0.8\left(\mathrm{~s}^{-1}\right)$ and $\omega=2 \pi(\mathrm{rad} / \mathrm{s})$, respectively. Theoretical solution to the problem can be found following the method presented in $[41,53]$, and the result is shown in Fig. 5 (see Appendix Appendix A).

It is revealed that inertia leads to a phase-lag in the velocity field with respect to the input strain rate. This makes the layers of the fluid flowing at different velocities and consequently results in a non-uniform flow across the channel. The lower the Reynolds number, the smaller the phase angle. Figure 6 presents the (strain-stress) Lissajous-Bowditch curves obtained for $R e=5 \times 10^{-4}$ to 5 . In Fig. $6, \Psi \approx 90^{\circ}$ for $R e=5 \times 10^{-4}$ while for higher Reynolds numbers, the phase angles of larger than $90^{\circ}$ are clearly detectable from the orientation of the Lissajous-Bowditch curves. Although introducing solid particles significantly disturbs the fluid flow, inertial effects are expected to be qualitatively similar. In order to keep the traces of inertia, in this article, $\left|G^{*}\right|$ and $\Psi$ are occasionally reported instead of $G^{\prime}$ and $\eta^{\prime}$. 


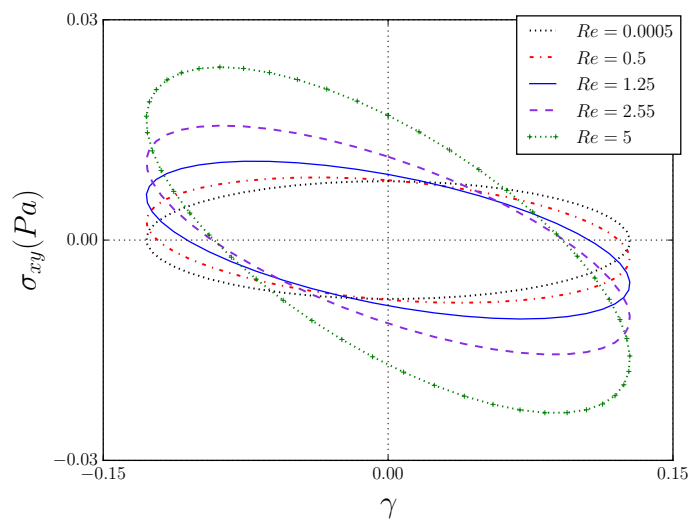

Figure 6: Lissajous-Bowditch curves obtained for a pure Newtonian fluid under an oscillatory shear test.

\section{Results}

In all test-cases solved in this paper, the radius of circular cylinders, $a=0.00125(m)$, and the height of the channel, $H=0.025(m)$, are kept constant. The solid bodies are initially vertically arranged with a gap equal to the discretization length, $\delta_{p}$; convergent solutions are obtained for $a / \delta_{p}=18.75$. In the following, the present SPH method is verified for a steady shear flow and then the viscoelastic response of the suspended magnetic chains is studied in an oscillatory shear flow.

\subsection{Verification}

The SPH solver is verified by studying the apparent shear viscosity obtained for a periodic array of magnetic chains with $N=8$ (see Fig. 1b) and $\chi=1$. The periodic length of the channel is $L=0.0125$, and the blockage ratio is $N(2 a) / H=0.8$. This problem was solved in [31], in a Stokes flow regime using a direct numerical simulation. In this steady problem, the inertial effects are insignificant for a relatively small particle Reynolds number, $R e_{p}=0.00625$.

Table 1 presents the non-dimensional average shear stress obtained using the SPH method in comparison with [31] for $M n=0.16$ and 0.016. Here, the effective polarization is $\hat{\beta}=0.25$. As discussed in [37], the SPH solution of a magnetostatic problem deviates from the finite-element solu- 
Table 1: The non-dimensional average shear stress $\sigma_{x y}^{*}=\sigma_{x y} / \dot{\gamma}_{0} \eta$ compared to the values reported in [31].

\begin{tabular}{c|c|c|c}
$M n$ & Kang et al. [31] & SPH & Error\% \\
\hline 0.16 & 2.704 & 2.697 & 0.28 \\
0.016 & 3.921 & 3.809 & 2.8
\end{tabular}

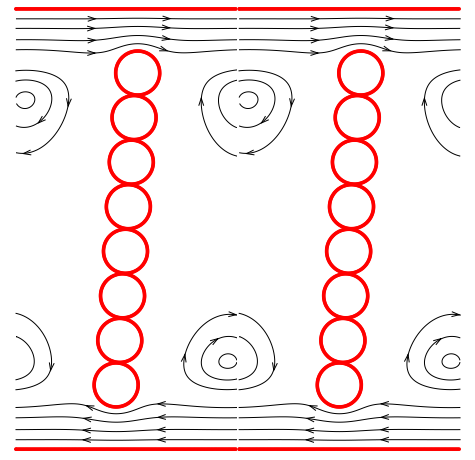

Figure 7: Stream-lines around a chain of magnetic solid particles under steady shear for $M n=0.016$.

tion by increasing either the external magnetic field or the magnetic susceptivity in the suspension. However, the solutions are in a good agreement for both cases. Figure 7 shows the steady-state streamlines around the magnetic chain for $M n=0.016$. In this figure, the computational domain is duplicated and the configuration of the system is illustrated for two adjacent magnetic chains.

\subsection{Oscillatory shear}

For the sake of comparison, geometry (see Fig. 1) is kept unchanged for the rest of results presented in this paper; $L=0.01(\mathrm{~m})$, and unless otherwise mentioned, the number of solid particles is $N=9$. The Reynolds number is changed by altering density and the Mason number is changed by adjusting the intensity of external magnetic field, while all other parameters are the same. In order to minimize the computational error, the magnetic susceptivity is set to $\chi=0.1[37]$. Unless otherwise mentioned, amplitude of the input strain is $\gamma_{0}=0.4 / \pi$ and its frequency is 


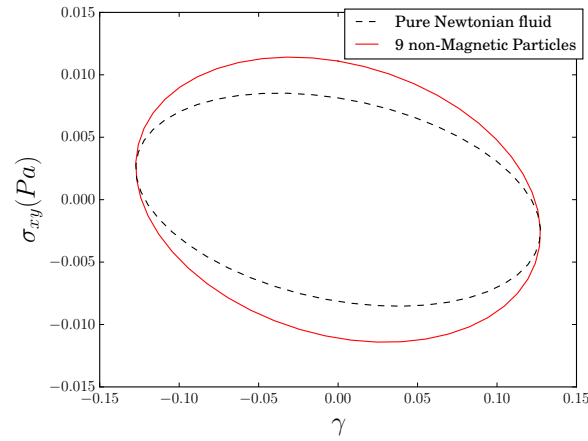

(a)

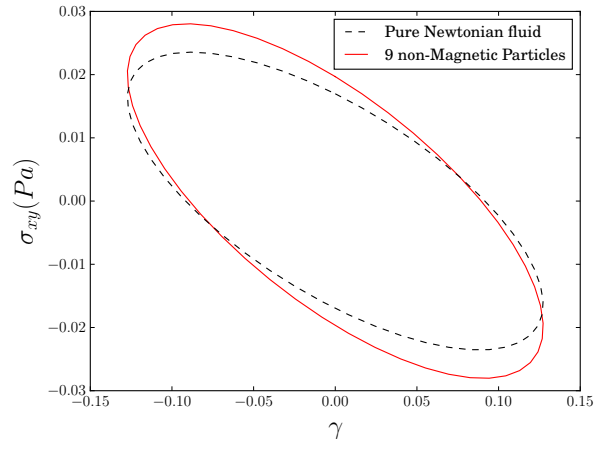

(b)

Figure 8: Lissajous-Bowditch curves in the absence of a magnetic field for (a) $R e=0.5$ and (b) $R e=5.0$ with $\gamma_{0}=0.4 / \pi$ and $\omega=2 \pi$.

$\omega=2 \pi(\mathrm{rad} / \mathrm{s})$. It should be noted that in the present work, for $\gamma_{0} \leq 0.13$, the stress response is almost linear; therefore, only the first harmonic response is investigated. As previously discussed, inertia substantially affects the computed apparent stress response in an oscillatory shear test. Therefore, it is useful to investigate first the effects of inertia for a non-magnetic suspension.

In Fig. 8, the Lissajous-Bowditch curves obtained for a suspension of non-magnetic particles are compared with cases of a pure Newtonian fluid. The curves are plotted for two different Reynolds numbers, $R e=0.5$ and 5 . It should be noted that for cases with suspended particles, the corresponding particle Reynolds numbers are $R e_{p}=0.00125$ and 0.0125 . The stress-strain Lissajous-Bowditch curve is a means to qualitatively investigate the stress response; the area confined by a curve is a measure of the energy lost in a cycle and for a linear stress response, the apparent storage modulus, $G^{\prime}$, is equal to the stress-strain slope $\frac{\left.\sigma_{x y}\right|_{\gamma=\gamma_{0}}}{\gamma_{0}}$.

As seen in Fig. 8 for both cases, the dissipated energy clearly increases by introducing suspended solid particles. This is due to the fact that solid particles are obstacles which further disturb the flow regime and add to the viscous dissipation. On the other hand, note that the stress-strain slope is almost unchanged for a rather small particle Reynolds number, $R e_{p}=0.00125$, for both with 


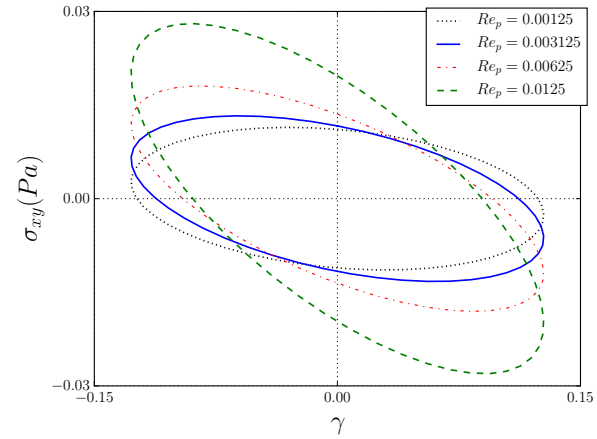

(a)

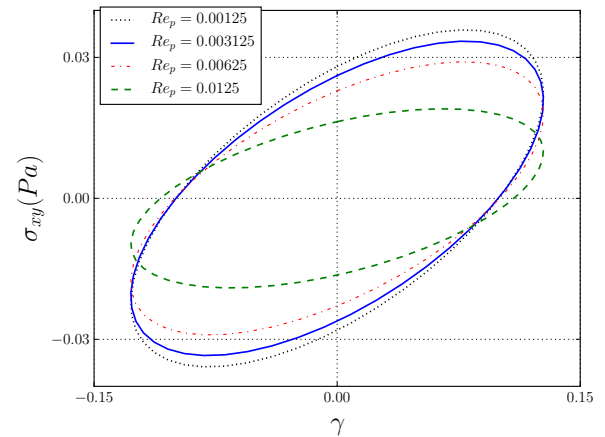

(b)

Figure 9: Lissajous-Bowditch curves obtained for (a) $M n \rightarrow \infty$ and (b) $M n=0.028$ with $\gamma_{0}=0.4 / \pi$ and $\omega=2 \pi$.

and without particles, while this is not the case for $R e=0.0125$. For a pure Newtonian fluid, the stress-strain slope is negative due to inertia. For a suspension in the absence of a magnetic field, solid particles are free to move with the suspending fluid; however, this movement is also governed by inertia due to their finite mass. The higher the particle Reynolds number, the more significant the effects of solid particles on apparent $G^{\prime}$. At the onset of applying an external magnetic field, depending on its strength, solid particles are not free anymore.

In Fig. 9, Lissajous curves are compared for both a zero magnetic field and $M n=0.028$ at different particle Reynolds numbers. While for all cases presented in Fig. 9a, the phase angle is $\pi / 2<\Psi$, a positive acute phase angle, $0<\Psi<\pi / 2$, is observed for all cases with $M n=0.028$ in Fig. 9b. For a rather small $M n$, the magnetized solid particles are attracted to each other forming a chain-like structure aligned with the field direction. Once the structure is deflected, induced magnetic forces tend to rotate the chain into its initial configuration. This works like a memory for the bulk system leading to an elastic response [52]. However, the time-scale of such a reconfiguration is limited by the hydrodynamic interactions which includes the viscous forces. The positive acute phase angle observed for $M n=0.028$, confirms that the magnetic suspension apparently acts as a viscoelastic medium. 


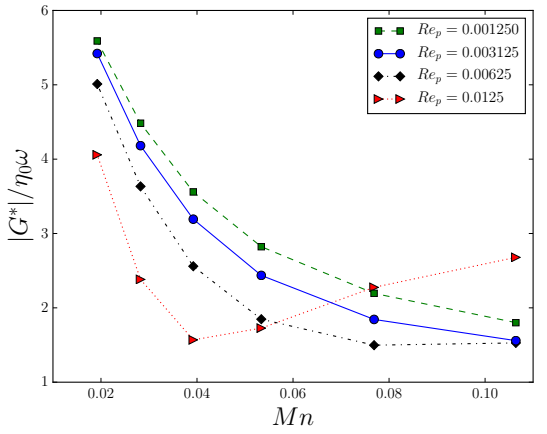

(a)

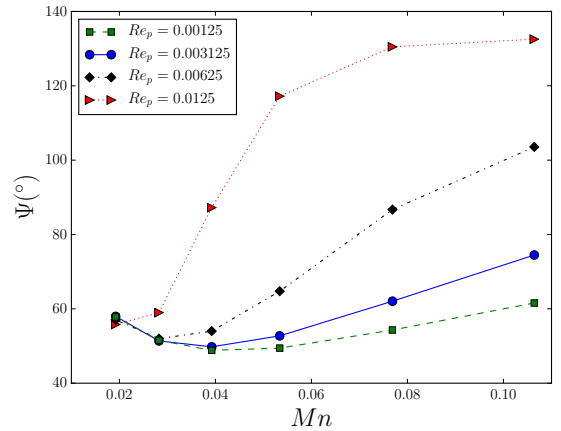

(b)

Figure 10: (a) The non-dimensional amplitude, $\left|G^{*}\right| / \eta_{0} \omega$, and (b) the phase angle, $\Psi$, of the apparent stress response as functions of $M n$ for different $R e_{p}, \gamma_{0}=0.4 / \pi$, and $\omega=2 \pi$.

By increasing the particle Reynolds number, the orientation of Lissajous-Bowditch curves are significantly changed as seen in Fig. 9a for a zero magnetic field intensity. The larger the particle Reynolds number, the larger the absolute value of the apparently negative $G^{\prime}$. Although $G^{\prime}$ decreases by increasing the Reynolds number also for $M n=0.028$, variation of the apparent modulus is more limited in Fig. 9b. This indicates the fact that magnetic forces suppress the inertial effects by restricting the chain-like structures. This issue is further investigated in Fig. 10 which presents the normalized amplitude and the phase angle of the apparent stress response as functions of the Mason number for different Reynolds numbers. As expected, by decreasing the Mason number, the apparent stress response is less sensitive to change in the Reynolds number. Also, the stronger the magnetic field, the larger the complex modulus, $\left|G^{*}\right|$. As discussed earlier, an obtuse phase angle is a definite sign of the dominance of inertia in an oscillatory shear test. For cases with $\Psi>\pi / 2$, the expected behaviour is reversed, i.e. $\left|G^{*}\right|$ becomes an increasing function of $M n$. In Fig. 10, the phase angle becomes obtuse at $M n \approx 0.04$ and 0.08 for $R e_{p}=0.0125$ and 0.00625 , respectively.

As seen in Fig. 10b for smaller Reynolds numbers, the phase angle shows a non-monotonic variation with $M n$. At a rather small Mason number, $M n<0.04$ for $R e_{p} \leq 0.003125$ in this 


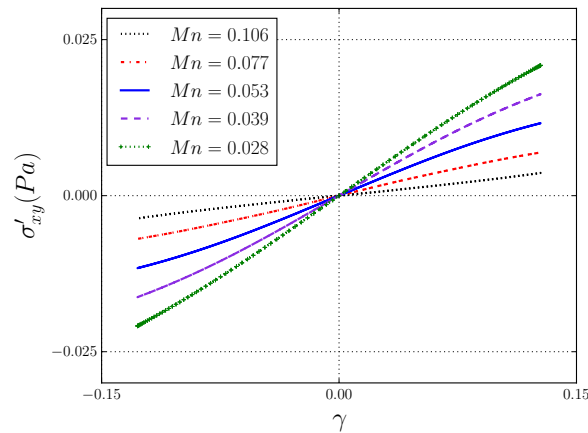

(a)

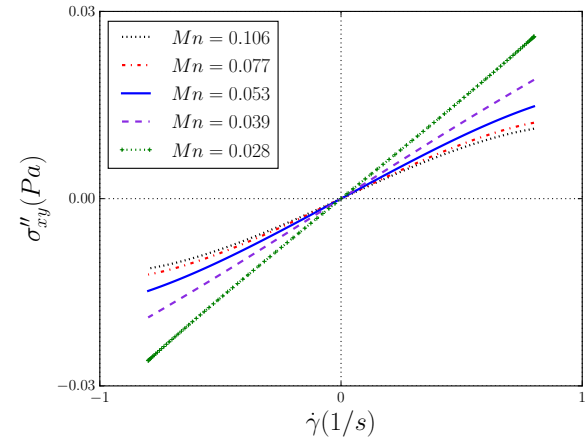

(b)

Figure 11: The intra-cycle variation of (a) the elastic stress and (b) the viscous stress obtained for $\operatorname{Re}_{p}=0.003125$, $\gamma_{0}=0.4 / \pi$, and $\omega=2 \pi$

work, $\Psi$ decreases by increasing the Mason number. Noting that inertial effects are weak in this range of magnetic field intensity, this means that viscous dissipation grows at a higher rate than elasticity of the system as the intensity of magnetic field increases. On the other hand, for a relatively larger Mason number, $M n>0.04$ in Fig. 10b, the phase angle is an increasing function of $M n$. Therefore, there is a maximum value for the ratio of elasticity to viscous dissipation in this system. By increasing the Reynolds number, the slope of $\Psi$ versus $M n$ significantly increases. This emphasizes the prominent role of inertia in weakening the apparent elasticity of the system. In a rather large Mason numbers for which $\Psi>\pi / 2$, the slope reduces and an asymptotic value is expected for $M n \rightarrow \infty$. Nevertheless, in all cases, the apparent viscosity dominates the viscoelastic behaviour of the system, i.e. $\pi / 4 \leq \Psi \leq 3 \pi / 4$. It should also be mentioned that for the present set of parameters, the quality of stress response is almost similar for $R e_{p}=0.00125$ and 0.003125 , so that in the following, for the sake of brevity some data are only shown for $R e_{p}=0.003125$.

In Fig. 11, the intra-cycle elastic and viscous stress responses are shown for $R e_{p}=0.003125$. Results shown in this figure include the third and fifth harmonics of the stress response in addition to the first harmonic. In Fig. 11a, the apparent elastic stress is almost linear for the whole range 


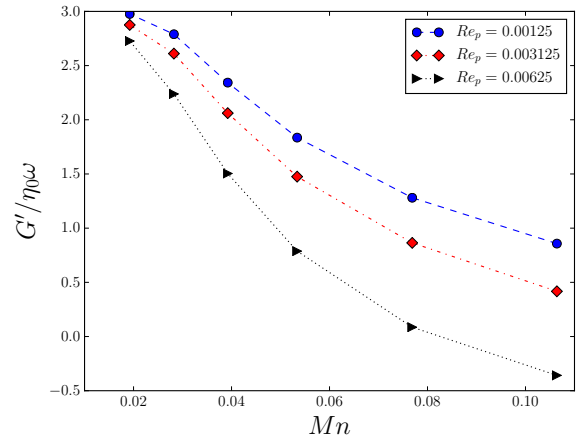

(a)

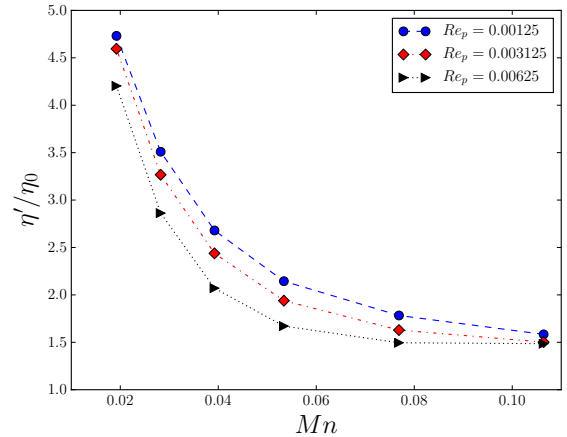

(b)

Figure 12: (a) The apparent elastic modulus and (b) the dynamic viscosity as functions of $M n$. Results are obtained for $R e_{p}=0.003125, \gamma_{0}=0.4 / \pi$, and $\omega=2 \pi$.

of $M n$, while the apparent viscous stress is slightly non-linear at rather large Mason numbers, $M n>0.07$ as seen in Fig. 11b. However, studying non-linearity of the stress response is outside the scope of this work. It can be seen in Fig. 11 that both the apparent elastic modulus and dynamic viscosity, defined as the slope in Figs 11a and 11b, respectively, are enhanced by decreasing the Mason number. The non-dimensional apparent moduli are shown in Fig. 12 for three Reynolds numbers.

As expected and discussed earlier, both $G^{\prime}$ and $\eta^{\prime}$ are decreasing functions of the Mason number. Nevertheless, the slope is larger for the apparent dynamic viscosity than elasticity in a rather small $M n$. Also, $G^{\prime}$ is considerably affected by inertia, specially for a rather large Mason number; the larger the Reynolds number, the smaller the apparent elastic modulus of the system. On the other hand, $\eta^{\prime}$ decreases only slightly when the Reynolds number increases. In Fig. 13, the apparent complex modulus and phase angle are shown as functions of the flux density of the external magnetic field for two different $\gamma_{0}$.

In Fig. 13, $\left|G^{*}\right|$ and $\Psi$ are shown as functions of $M n^{*}$ for two strain amplitudes. Keeping the frequency of oscillations constant, $\dot{\gamma}_{0}$ is proportional to $\gamma_{0}$. In this manner, according to Eq. (8), 


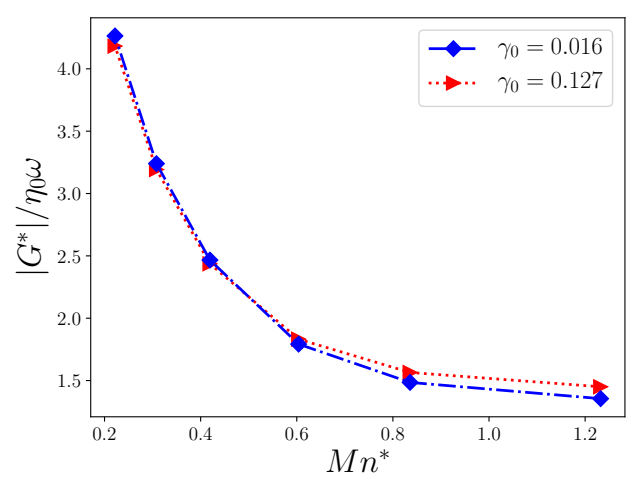

(a)

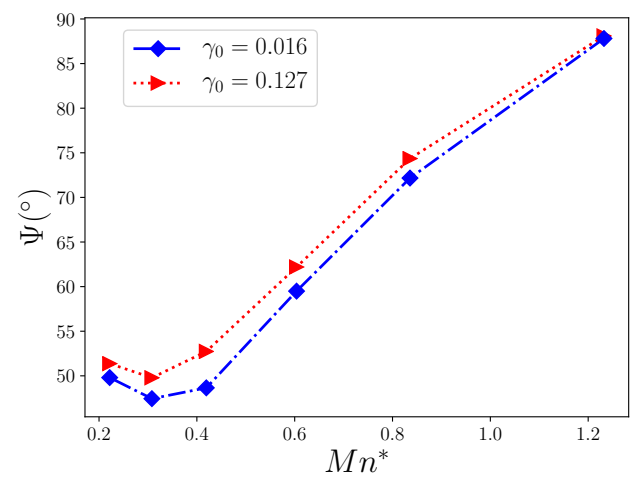

(b)

Figure 13: (a) The non-dimensional amplitude, $\left|G^{*}\right| / \eta_{0} \omega$, and (b) the phase angle, $\Psi$, of the apparent stress response as functions of $M n^{*}$ for two different $\gamma_{0}, R e_{p}=0.00125\left(\gamma_{0}=0.127\right)$ and $R e_{p}=0.00039\left(\gamma_{0}=0.016\right)$, and $\omega=2 \pi$.

the (conventional) Mason number is also proportional to $\gamma_{0}$ for a fixed $B_{0}$ (or equivalently a fixed $\left.M n^{*}\right)$. The interesting point is that in Fig. $13,\left|G^{*}\right|$ and $\Psi$ are almost the same for both $\gamma_{0}$ values while the corresponding values of $M n$ are eight-times different.

\subsubsection{Particles configuration}

A simplified physical model for a suspension of magnetic solid particles is considered in the present work, and its apparent viscoelastic response was studied earlier in this paper. However, it is also important to investigate the micro-structure of suspended magnetic chains and realize its link with the apparent rheology of the system. In cases of gap-spanning magnetic chains, it is fairly reasonable to assume an affine micro-structural deformation [24]. On the other hand, there is certainly a phase angle between an oscillatory strain and a deforming non-gap-spanning microstructure. This phase angle is a function of the magnetic field intensity and the Reynolds number. Time-history of the normalized input strain, apparent shear stress, and coordinate of solid particle $P_{1}$ positioned at the bottom of a magnetic chain is illustrated in Fig. 14 for $R e_{p}=0.003125$ with and without an external magnetic field. The spatial position of $P_{1}$ can be considered as an indicator of deflection for the magnetic chain. 


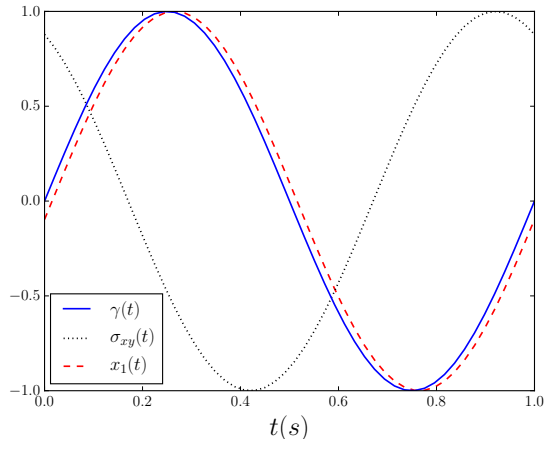

(a)

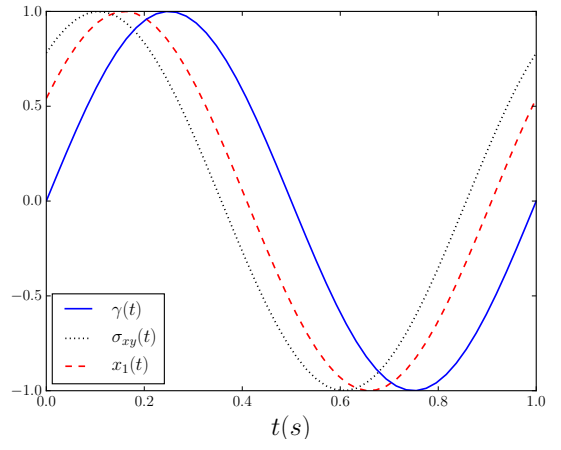

(b)

Figure 14: The time-history of the normalized input strain, apparent stress, and the position of $P_{1}$. Results are obtained for (a) $M n \rightarrow \infty$ and (b) $M n=0.039$, with $R e_{p}=0.003125, \gamma_{0}=0.4 / \pi$, and $\omega=2 \pi$.

For the non-magnetic case presented in Fig. 14a, the movement of solid particles is almost synchronized with the input strain; however, there is a small phase-lag due to inertia, while the apparent stress is completely out of phase, i.e. $\Psi>\pi / 2$. On the contrary for $M n=0.039$, particle movement has a leading phase with respect to the input strain as observed in Fig. 14b. Furthermore, the strain-stress phase angle, $\Psi$, decreases significantly for $M n=0.039$ in comparison with the non-magnetic case. Figure 15 presents the phase angles of the chain deflection, $\Psi^{x-\gamma}$ and $\Psi^{\sigma_{x y}-x}$, calculated for location of $P_{1}$ with respect to the input strain and the apparent stress, respectively. These phase angles are obtained for different Reynolds numbers, and illustrated as functions of $\mathrm{Mn}^{-1}$. Here, the inverse of the Mason number is used to be able to include results obtained for $B_{0}=0(M n \rightarrow \infty)$.

For all Reynolds numbers, $\Psi^{x-\gamma}$ is a monotonically increasing function of $M n^{-1}\left(B_{0}\right)$. The smaller the Reynolds number, the larger the strain-deflection phase angle. For $B_{0}=0(M n \rightarrow \infty)$ with a rather small $R e_{p}$, an almost synchronized deflection is observed, while a phase-lag is caused by inertia, i.e. $\Psi^{x-\gamma} \approx 0$ for $R e_{p}=0.00125$ and $\Psi^{x-\gamma}<0$ for larger Reynolds numbers. On the other hand, $\Psi^{\sigma_{x y}-x}$ is a decreasing function of $M n^{-1}$. The larger the Reynolds number, the 


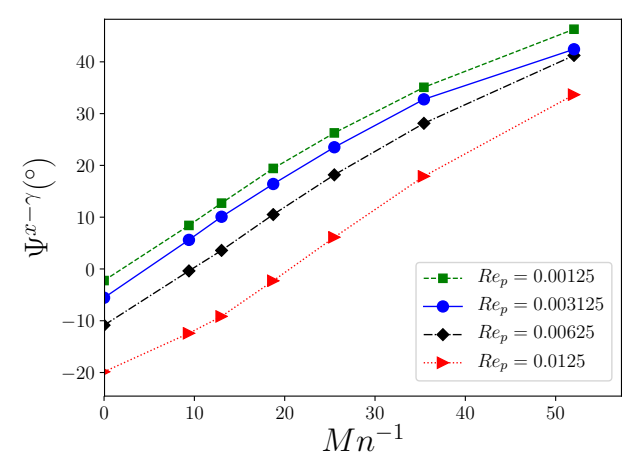

(a)

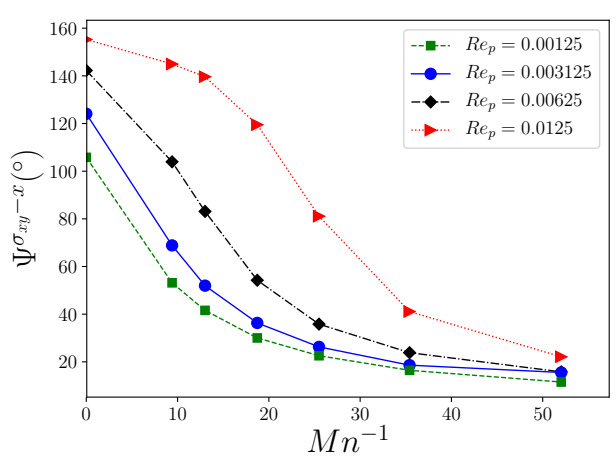

(b)

Figure 15: (a) The strain-deflection phase angle, $\Psi^{x-\gamma}$, and (b) the deflection-stress phase angle, $\Psi^{\sigma_{x y}-x}$, as functions of $M n^{-1}$, obtained for $\gamma_{0}=0.4 / \pi$, and $\omega=2 \pi$.

larger the deflection-stress phase angle, and $\Psi^{\sigma_{x y}-x}$ is less sensitive to the Reynolds number for a rather large $M n^{-1}$. The interesting point in Fig. 15 is that, although $\Psi^{x-\gamma}$ varies significantly with $M n^{-1}$, its variation with the Reynolds number is almost the same for all magnetic flux densities. This shows that inertia affects the time-history of the motion of solid particles almost independent of the imposed magnetic field. Configurations of the whole magnetic chain coinciding with $\gamma=0$ and $\gamma=\gamma_{0}$ are shown in Fig. 16, where $\hat{x}=(x-L / 2) /(L / 2)$.

Knowing that the motion of non-magnetic solid particles is almost in-line with the input strain for $R e_{p}=0.00125$, a phase-lead in the deflection of magnetic chain is clearly observed with respect to $\gamma$, in Fig. 16a. In Fig. 16b, the non-magnetic solid particles are almost located at their farthest position for $\gamma=\gamma_{0}$. For larger Reynolds numbers, solid particles exhibit a similar behaviour; however, with slightly different phase angles (see Fig. 15a) that are hardly detectable in Fig. 16. In all cases, solid particles are maintained in a chain-like structure and the configuration of the system does not deviate from model illustrated in Fig. 1. In order to further compare the motion of solid particles for different sets of parameters, a normalized local chain slope is defined as

$$
\bar{s}=\frac{1}{\gamma_{0}} \frac{x-\frac{L}{2}}{y-\frac{H}{2}}
$$




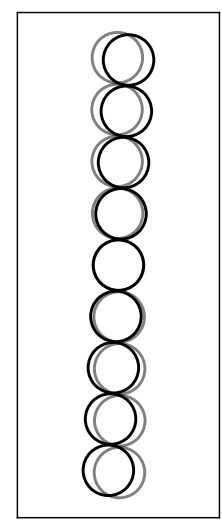

(a) $\gamma=0$

Light: $\hat{x}_{1} \approx 0.01$

Dark: $\hat{x}_{1} \approx-0.1$

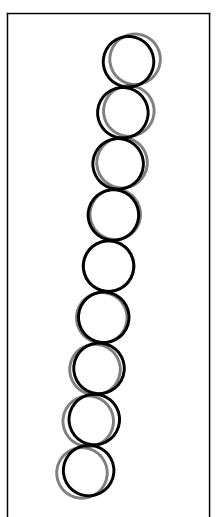

(b) $\gamma=\gamma_{0}$

Light: $\hat{x}_{1} \approx-0.26$ Light: $\hat{x}_{1} \approx 0.03$

Dark: $\hat{x}_{1} \approx-0.2 \quad$ Dark: $\hat{x}_{1} \approx-0.09$

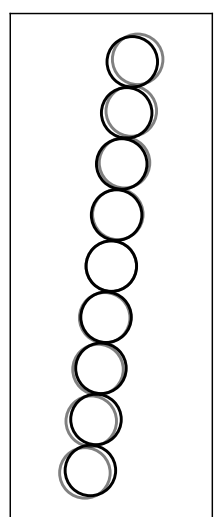

(d) $\gamma=\gamma_{0}$

Light: $\hat{x}_{1} \approx-0.26$

Dark: $\hat{x}_{1} \approx-0.21$

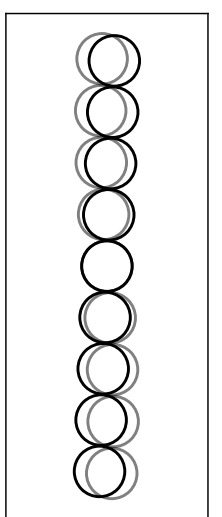

(e) $\gamma=\gamma_{0}$

Light: $\hat{x}_{1} \approx 0.05$

Dark: $\hat{x}_{1} \approx-0.07$

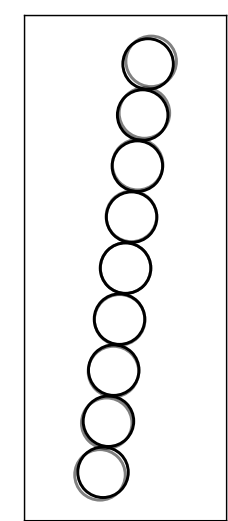

(f) $\gamma=\gamma_{0}$

Light: $\hat{x}_{1} \approx-0.25$ Dark: $\hat{x}_{1} \approx-0.22$

Figure 16: Position of solid particles obtained for (a,b) $R e_{p}=0.00125$, (c,d) 0.003125, and (e,f) 0.00625, with $\omega=2 \pi$ and $\gamma_{0}=0.4 / \pi$. Lighter circles are the results for $M n \rightarrow \infty$, while dark ones belong to the case with $M n=0.039$.

and is presented in Fig. 17 for the four bottom solid particles in a chain at the time when $P_{1}$ is at its extreme lateral position. For the non-magnetic case presented in Fig. 17a, the maximum chain slope increases by reducing the Reynolds number; for $R e=0.00125$, the extent of displacement is slightly larger than that of the channel walls. This trend is reversed for the magnetic case illustrated in Fig. 17b; the smaller the Reynolds number, the smaller the maximum chain slope. It is deduced from Fig. 17 that the imposition of an external magnetic field reduces the extent of displacement of the solid particles. Nevertheless, this limitation on the motion of solid particles is relaxed by increasing the Reynolds number. Therefore, it can be inferred that the effectiveness of the magnetic field on the extent of motion of solid particles is decreased by inertia. Another point is that inertia has a more significant effect on the local chain slope nearer to the centre of a chain. An interesting point in Fig. 17 is the changing curvature of the graphs. This is because in Fig. 17b, there is a magnetic bond between solid particles which resists their relative motion due to different hydrodynamic forces. 


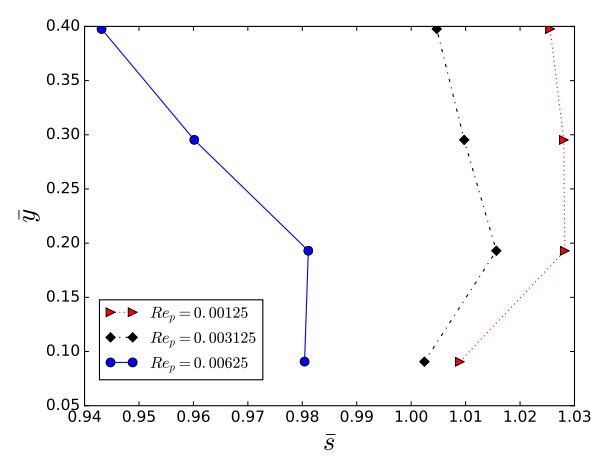

(a)

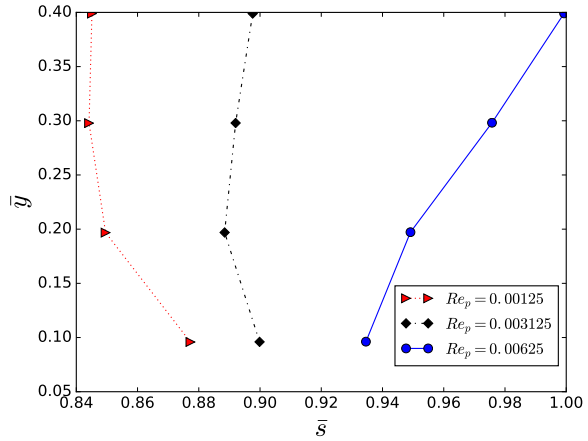

(b)

Figure 17: The normalized local chain slope for the four bottom solid particles with $P_{1}$ at its extreme lateral position. Results are obtained for $\omega=2 \pi, \gamma_{0}=0.4 / \pi$, and (a) $M n \rightarrow \infty$ and (b) $M n=0.039$.

\subsubsection{Blockage Ratio}

So far in this paper, the stress response was computed for $N=9$, resulting in a blockage ratio of $N(2 a) / H=0.9$. Figure 18 presents the amplitude and phase angle of the computed stress response for different number of solid particles (blockage ratios). The results are shown as functions of the Mason number. It is evident that the trends are similar for all $N$ numbers, i.e. $\left|G^{*}\right|$ decreases by increasing $M n$, while $\Psi$ is an increasing function of the Mason number. Nevertheless, by increasing the blockage ratio, the magnetic cluster can exert a more effective resistance against fluid flow in the channel, which leads to

- a decrease in $\Psi$, as it decreases the effect of inertia and increases the effect of elasticity

- a stronger stress response

- more significant variations in $\left|G^{*}\right|$ and $\Psi$ as functions of $M n$

In the rest of this work, the blockage ratio is set to 0.9 in order to maintain the significance of the viscoelastic response of the model. 


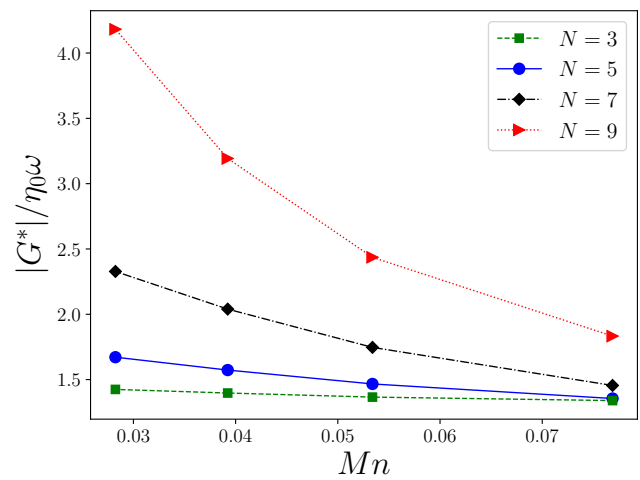

(a)

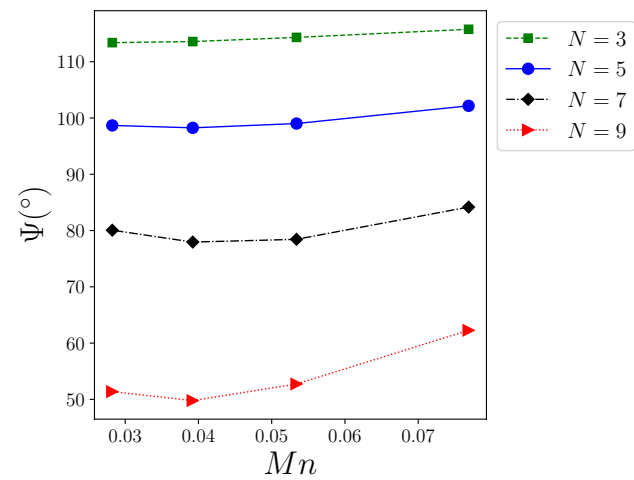

(b)

Figure 18: (a) The non-dimensional amplitude, $\left|G^{*}\right| / \eta_{0} \omega$, and (b) the phase angle, $\Psi$, of the computed stress response as functions of $M n$ for different $N$, with $\gamma_{0}=0.4 / \pi$, and $\omega=2 \pi$.

\subsubsection{Effects of frequency}

In the previous section, the frequency of the input strain was kept constant, $\omega=2 \pi$. However, generally for a viscoelastic material, moduli are functions of frequency [52]. For magnetorheological fluids, such a functionality has been addressed in the literature $[18,24,54]$. Frequency is an important factor which affects the micro-structure and consequently alters the rheology. Furthermore, in determining the significance of inertia in a dynamic shear test, frequency is an important factor along with the size of the test domain $[55,56]$. Keeping all parameters constant, the higher the

frequency, the more significant the inertial effects. In this sense, the Stokes number, $S t=\left|\frac{\rho \omega H^{2}}{G^{*}}\right|$, is introduced as a measure of the significance of inertia $[55,56]$. It is worth to note that, the criterion reported in the literature [56] for omitting inertial effects from apparent complex modulus, is not applicable to the suspension of magnetized solid particles.

In an oscillatory shear test, the strain rate and strain are directly related to frequency, $\dot{\gamma}_{0}=\omega \gamma_{0}$. Therefore, among three input parameters, $\dot{\gamma}_{0}, \gamma_{0}$, and $\omega$, there are only two independent variables. In the first set of test-cases, $\dot{\gamma}_{0}=0.8(1 / s)$ is kept constant and $\omega$ varies. Results are shown as Lissajous-Bowditch curves in Fig. 19 for $M n=0.053$ and different Reynolds numbers. In these 


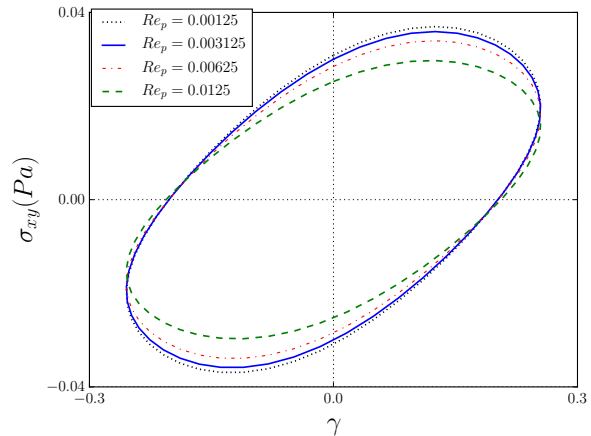

(a)

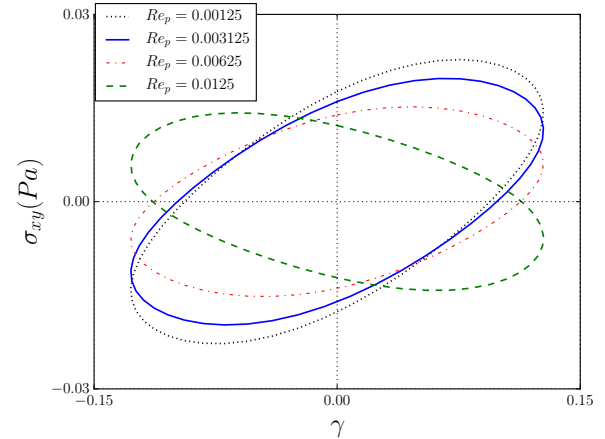

(b)

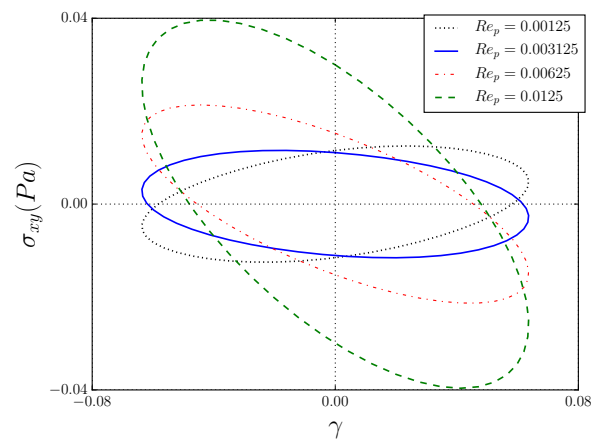

(c)

Figure 19: Lissajous-Bowditch curves obtained for $\dot{\gamma}_{0}=0.8(1 / s), M n=0.053$, and different Reynolds numbers, with (a) $\omega=\pi$, (b) $\omega=2 \pi$, and (c) $\omega=4 \pi$.

test-cases, the smaller the frequency the larger the strain amplitude; thus a slightly non-linear response is observed in Fig. 19a. Nevertheless, it is clear that for $\omega=4 \pi$, inertia dramatically affects the result, while for $\omega=\pi$, the stress response is less sensitive to the Reynolds number. Stokes numbers obtained for the apparent complex moduli, $\left|G^{*}\right|$, corresponding to $R e_{p}=0.003125$ and $\omega=\pi, 2 \pi$, and $4 \pi$ are $S t=1.4,5$, and 17, respectively. As observed in Fig. 19, results are qualitatively similar for $S t \sim O(1)$.

In the following, $\gamma_{0}=0.4 / \pi$ is kept constant and since $\dot{\gamma}_{0}$ varies with frequency, both of the non-dimensional numbers, $M n$ and $M n^{*}$, vary for a constant external magnetic field. In this sense, 


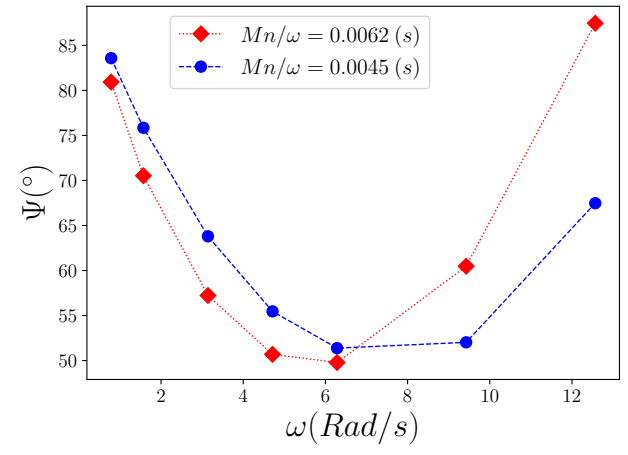

(a)

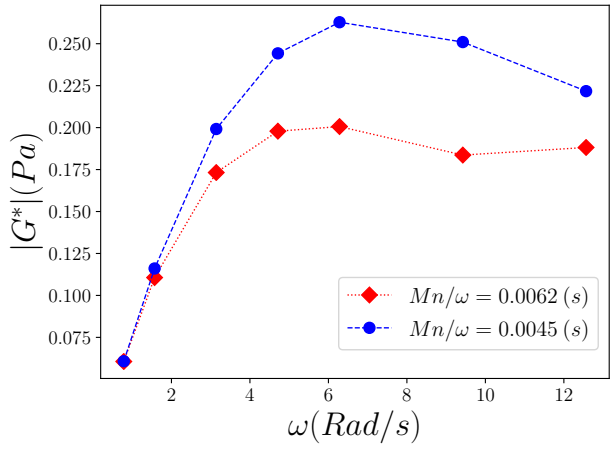

(b)

Figure 20: (a) The magnitude of the complex modulus, $\left|G_{1}^{*}\right|$, and (b) the phase angle, $\Psi_{1}$, of the apparent stress response as functions of $\omega$ for $R e_{p}=0.003125, \gamma_{0}=0.4 / \pi$, and two different Mason numbers.

$M n / \omega$ is used as a measure of the strength of the external magnetic field. Presented in Fig. 20 are the results obtained for $R e_{p}=0.003125$ and two different magnetic flux densities. In these test-cases with $\omega=2 \pi$, the Stokes numbers are $S t=3.8$ and 2.9 for $M n / \omega=0.0062(s)$ and $M n / \omega=0.0045(s)$, respectively. By increasing frequency, inertial effects become more significant and based on the above discussions, $\left|G^{*}\right|$ decreases while $\Psi$ is an increasing function of frequency. The maximum value for $\left|G^{*}\right|$ and the minimum phase angle are observed for $\omega \approx 2 \pi$.

As observed in Fig. 20 for $\omega \leq 2 \pi,\left|G^{*}\right|$ is an increasing function of $\omega$, while $\Psi$ is decreasing. In both Figs. 20a and 20b, the slope gradually decreases by increasing $\omega$. For $\omega>2 \pi$, inertia causes a decrease in $\left|G^{*}\right|$ and a sharp increase in the phase angle. The frequency dependent behaviour of the system is further studied in Fig. 21 that presents the intra-cycle variation of $\sigma_{x y}^{\prime}$ and $\sigma_{x y}^{\prime \prime}$. The stress response is almost linear for all cases shown in Fig. 21; however, by keeping $\gamma_{0}=0.4 / \pi$ constant and increasing $\omega$, the stress response becomes slightly non-linear. This nonlinearity is more significant for a weaker magnetic field. This is due to the fact that for a constant $\gamma_{0}$, frequency is directly proportional to $\dot{\gamma}_{0}$, and consequently, hydrodynamic forces are enhanced. This leads to a larger chain deformation, as discussed later in this paper, and higher nonlinearities. 


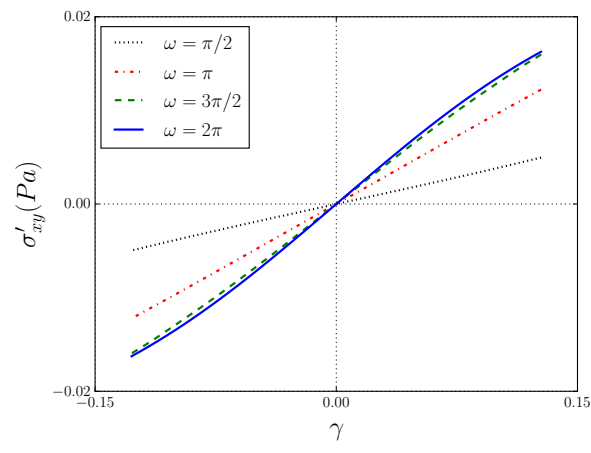

(a)

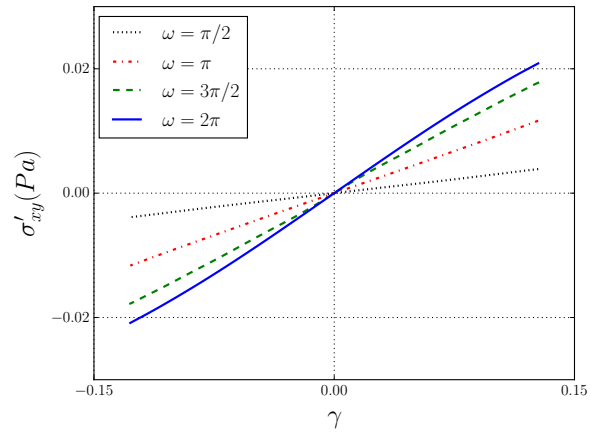

(c)

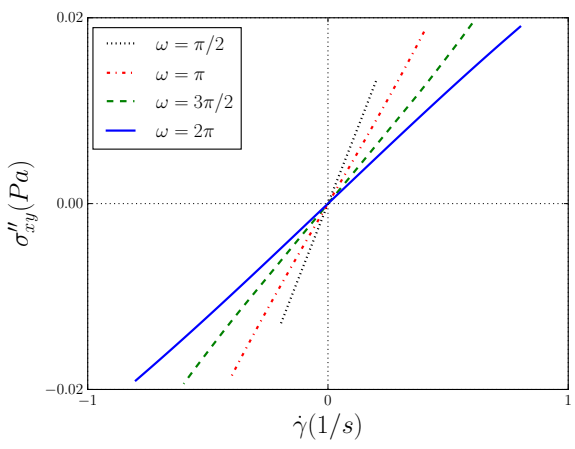

(b)

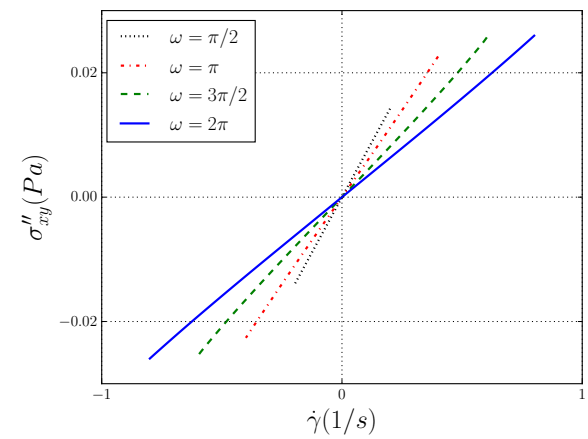

(d)

Figure 21: The intra-cycle variation of the elastic stress (a,c) and the viscous stress (b,d) obtained for $\operatorname{Re}_{p}=0.003125$, and $\gamma_{0}=0.4 / \pi$. The external magnetic flux density is $M n / \omega=0.0062(s)$ for (a) and (b), while for (c) and (d) it is $M n / \omega=0.0045(s)$. 


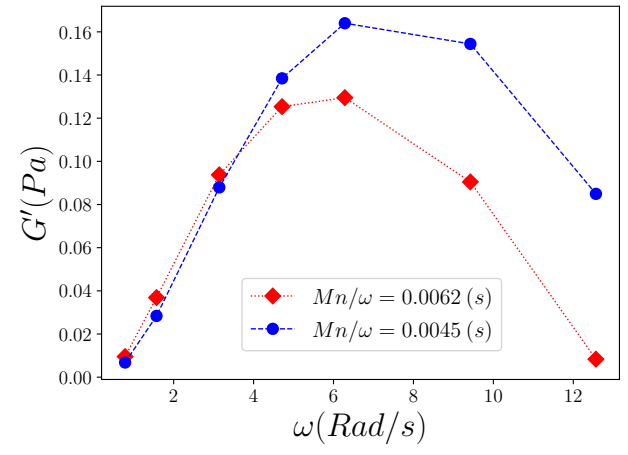

(a)

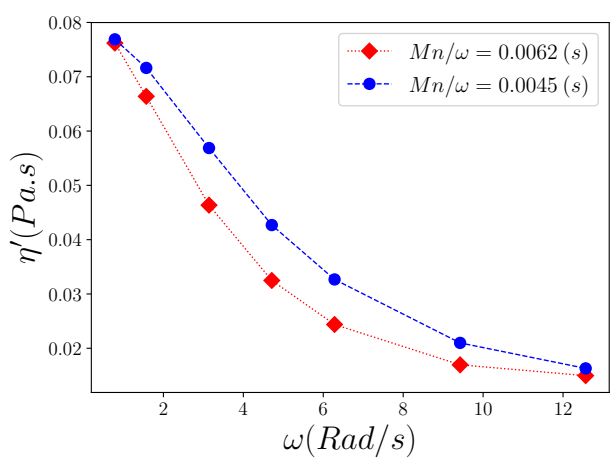

(b)

Figure 22: (a) The apparent elastic modulus and (b) the dynamic viscosity as functions of $\omega$. Results are obtained for $R e_{p}=0.003125, \gamma_{0}=0.4 / \pi$, and two different magnetic flux densities.

It is evident in Fig. 21 that by increasing frequency, the storage modulus increases while dynamic viscosity decreases for both $M n / \omega=0.0062(s)$ and $M n / \omega=0.0045(s)$. This is quantitatively shown in Fig. 22 where $G^{\prime}$ and $\eta^{\prime}$ are shown as functions of $\omega$. Here, the results are also shown for $\omega>2 \pi$ where a dramatic inertial effect leads to a decrease in $G^{\prime}$. Nevertheless, as discussed earlier, the apparent dynamic viscosity is less sensitive to inertia. These results can be compared with similar behaviour reported in the literature $[20,54]$ for a magnetorheological fluid under a dynamic shear load with a rather large amplitude and high frequency.

In Fig. 23, magnetized solid particles are shown forming magnetic chains for the current set of parameters. In this figure, the configurations of chains are compared for different frequencies with $\gamma=0$ and $\gamma=\gamma_{0}$, along with the maximum deflection. At the starting of a cycle, magnetic chains are tilted almost to the same extent for all three frequencies as shown in Fig. 23a. During a cycle for a constant $\gamma_{0}$, the amplitude of the imposed strain rate and consequently, the magnitude of the hydrodynamic force (mostly viscous force due to a small $R e_{p}$ ) are greater for a higher frequency. Therefore, the magnetic chain is tilted more for $\omega=2 \pi$ in Figs. 23b and 23c. The more the chain is tilted, the higher the amount of energy stored in the system for a constant external magnetic 


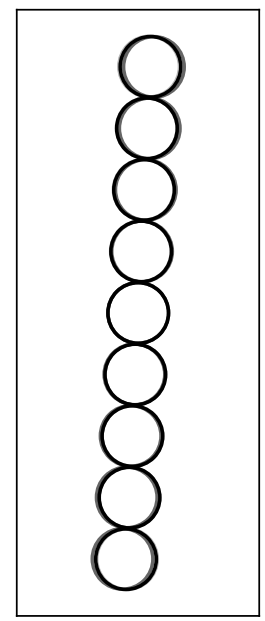

(a)

Lightest: $\hat{x}_{1} \approx-0.09$

Light: $\hat{x}_{1} \approx-0.13$

Dark: $\hat{x}_{1} \approx-0.1$

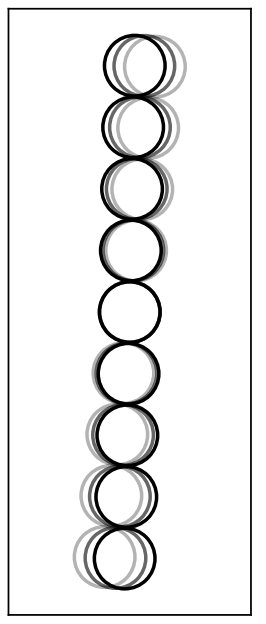

(b)

Lightest: $\hat{x}_{1} \approx-0.21$

Light: $\hat{x}_{1} \approx-0.12$

Dark: $\hat{x}_{1} \approx-0.04$

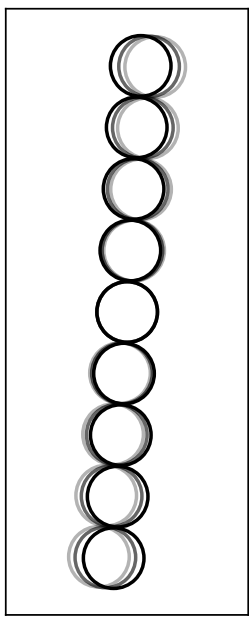

(c)

Lightest: $\hat{x}_{1} \approx-0.23$

Light: $\hat{x}_{1} \approx-0.18$

Dark: $\hat{x}_{1} \approx-0.11$

Figure 23: Position of solid particles obtained for $M n / \omega=0.0062(s), R e_{p}=0.003125$ and $\gamma_{0}=0.4 / \pi$ with (a) $\gamma=0$, (b) $\gamma \approx \gamma_{0}$, and (c) once the chain reaches its maximum deflection. Results obtained for $\omega=2 \pi$ are shown with the lightest circles while darker ones depict the cases with $\omega=\pi$ and $\pi / 2$, respectively. 


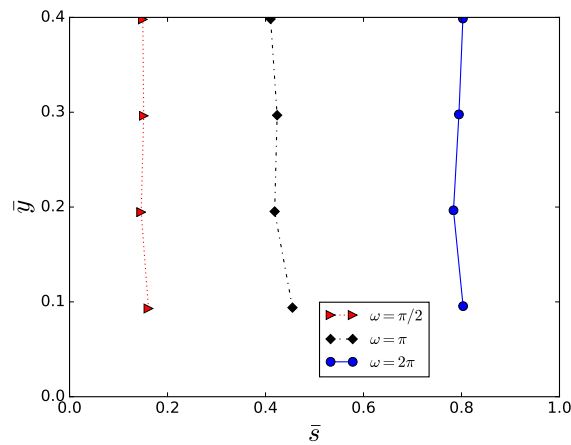

(a)

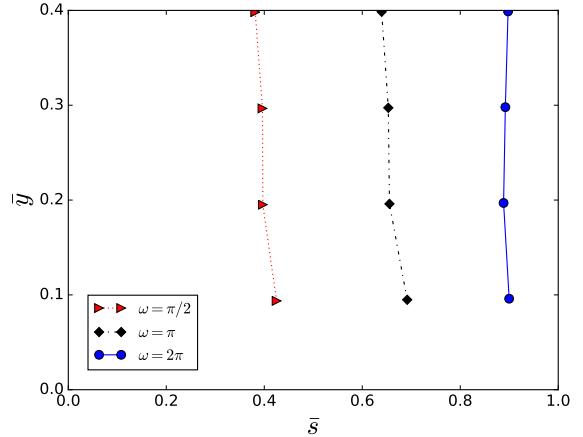

(b)

Figure 24: The normalized local chain slope for the four bottom solid particles in a chain at (a) $\gamma \approx \gamma_{0}$ and (b) the maximum chain deflection, obtained for $M n / \omega=0.0045(s)$ and $\gamma_{0}=0.4 / \pi$.

field intensity. This leads to a larger storage modulus and a smaller phase angle. Also, a larger chain tilting and consequently a wider gap result in a reduction in the loss of energy by viscous dissipation. In Fig. 24, the local chain slope for the four bottom solid particles are shown. By increasing the frequency, not only the maximum chain slope increases but also $\bar{s}$ has a smaller variation from the instant of $\gamma=\gamma_{0}$ to its maximum value. It can be inferred from Fig. 24 that the chain-like structures keep an almost straight shape during a small amplitude oscillatory shear test.

\section{Conclusion}

In this paper, the rheological behaviour of a periodic suspension of vertically arranged paramagnetic solid particles was numerically investigated using oscillatory shear tests at finite Reynolds numbers. The non-gap-spanning chains of solid particles were formed upon the imposition of an external magnetic field, and hence, the system behaved like a viscoelastic fluid with the ratio of elasticity to viscous dissipation being higher than unity, i.e. $\omega \eta^{\prime}>G^{\prime}$. The results of this work are summarized in Tables 2 and 3 for cases with a relatively large inertia and a rather strong magnetic field, respectively. It can also be added that 
Table 2: A summary of results with significant inertial effects.

\begin{tabular}{|l|c|c|c|c|c|}
\hline & $\left|G^{*}\right|$ & $\Psi$ & Apparent $G^{\prime}$ & Viscous dissipation & Chain tilting \\
\hline Presence of particles & $\uparrow$ & $\uparrow$ & $\begin{array}{c}\text { cte }\left(\text { negligible } R e_{p}\right) \\
\downarrow\left(\text { finite } R e_{p}\right)\end{array}$ & $\uparrow$ & - \\
\hline Increasing inertia & $\uparrow$ & $\uparrow$ & $\downarrow$ & $\uparrow$ & slightly $\downarrow$ \\
\hline Strengthening magnetics & $\downarrow$ & $\downarrow$ & $\uparrow$ & $\downarrow$ & $\downarrow$ \\
\hline Increasing frequency (constant $\left.\gamma_{0}\right)$ & slightly $\downarrow$ & $\uparrow$ & $\downarrow$ & $\downarrow$ & $\uparrow$ \\
\hline
\end{tabular}

Table 3: A summary of results for a relatively strong magnetic field.

\begin{tabular}{|l|c|c|c|c|c|}
\hline & $\left|G^{*}\right|$ & $\Psi$ & Elasticity & Viscous dissipation & Chain tilting \\
\hline Increasing inertia & $\downarrow$ & $\uparrow$ & $\downarrow$ & slightly $\downarrow$ & $\uparrow$ \\
\hline Strengthening magnetics & $\uparrow$ & $\downarrow$ & $\uparrow$ & $\uparrow$ & $\downarrow$ \\
\hline Increasing frequency (constant $\gamma_{0}$ ) & $\uparrow$ & $\downarrow$ & $\uparrow$ & $\downarrow$ & $\uparrow$ \\
\hline
\end{tabular}

- For a rather small Mason number, the ratio of elasticity to viscous dissipation decreased by increasing the intensity of the external magnetic field.

- For the current system, the phase-lag imposed by inertia in the movement of solid particles with respect to the input strain, gradually decreased by increasing the strength of the magnetic field and ultimately switched to a phase-lead.

- The higher the frequency at a constant shear rate, the stronger the inertial effects.

- For a rather small Stokes number, the system behaved as expected from a viscoelastic system similar to a spring and a damper in series, i.e. elasticity increased while viscosity decreased by increasing frequency.

- In all cases, viscous dissipation was a decreasing function of frequency and was only slightly affected by inertia, while the calculated elasticity was substantially a decreasing function of inertia.

Conducting similar studies for a wider range of concentration, blockage ratio, magnetic flux density, 
and the Reynolds number, one might be able to define a proper constitutive equation for MR fluids in the post-yield state.

\section{References}

[1] M. R. Jolly, J. W. Bender, J. D. Carlson, Properties and applications of commercial magnetorheological fluids, in: 5th Annual International Symposium on Smart Structures and Materials, International Society for Optics and Photonics, 1998, pp. 262-275.

[2] A.-G. Olabi, A. Grunwald, Design and application of magneto-rheological fluid, Materials \& design 28 (10) (2007) 2658-2664.

[3] M. R. Jolly, J. D. Carlson, B. C. Munoz, A model of the behaviour of magnetorheological materials, Smart Materials and Structures 5 (5) (1996) 607.

[4] J. D. Carlson, M. R. Jolly, MR fluid, foam and elastomer devices, mechatronics 10 (4) (2000) $555-569$.

[5] W. Li, P. Zhang, X. Gong, P. Kosasih, Linear viscoelasticity of MR fluids: dependence on magnetic fields, International Journal of Modern Physics B 19 (07n09) (2005) 1198-1204.

[6] S. S. Deshmukh, G. H. McKinley, Rheological behavior of magnetorheological suspensions under shear, creep and large amplitude oscillatory shear (laos) flow, Proc. XIVth Int. Congr. on Rheology.

[7] E. Lemaire, Y. Grasselli, G. Bossis, Field induced structure in magneto and electro-rheological fluids, Journal de Physique II 2 (3) (1992) 359-369.

[8] M. T. López-López, P. Kuzhir, J. Caballero-Hernandez, L. Rodríguez-Arco, J. D. Duran, G. Bossis, Yield stress in magnetorheological suspensions near the limit of maximum-packing fraction, Journal of Rheology (1978-present) 56 (5) (2012) 1209. 
[9] J. Ginder, L. Davis, L. Elie, Rheology of magnetorheological fluids: Models and measurements, International journal of modern physics b 10 (23n24) (1996) 3293-3303.

[10] J. de Vicente, M. T. López-López, J. D. Durán, F. González-Caballero, Shear flow behavior of confined magnetorheological fluids at low magnetic field strengths, Rheologica acta 44 (1) (2004) 94-103.

[11] J. Segovia-Gutiérrez, C. Berli, J. De Vicente, Nonlinear viscoelasticity and two-step yielding in magnetorheology: A colloidal gel approach to understand the effect of particle concentration, Journal of Rheology (1978-present) 56 (6) (2012) 1429-1448.

[12] D. Kittipoomwong, D. J. Klingenberg, J. C. Ulicny, Dynamic yield stress enhancement in bidisperse magnetorheological fluids, Journal of Rheology (1978-present) 49 (6) (2005) 15211538.

[13] J. Claracq, J. Sarrazin, J.-P. Montfort, Viscoelastic properties of magnetorheological fluids, Rheologica Acta 43 (1) (2004) 38-49.

[14] W. Li, H. Du, N. Guo, Dynamic behavior of MR suspensions at moderate flux densities, Materials Science and Engineering: A 371 (1) (2004) 9-15.

[15] X. Wang, F. Gordaninejad, Flow analysis of field-controllable, electro-and magneto-rheological fluids using herschel-bulkley model, Journal of Intelligent Material Systems and Structures 10 (8) (1999) 601-608.

[16] J. Ramos, J. de Vicente, R. Hidalgo-Alvarez, Small-amplitude oscillatory shear magnetorheology of inverse ferrofluids, Langmuir 26 (12) (2010) 9334-9341.

[17] L. J. Felicia, J. Philip, Probing of field-induced structures and their dynamics in ferrofluids using oscillatory rheology, Langmuir 30 (41) (2014) 12171-12179. 
[18] W. Li, G. Chen, S. Yeo, Viscoelastic properties of MR fluids, Smart Materials and Structures 8 (4) (1999) 460.

[19] K. D. Weiss, J. D. Carlson, D. A. Nixon, Viscoelastic properties of magneto-and electrorheological fluids, Journal of Intelligent Material Systems and Structures 5 (6) (1994) 772-775.

[20] W. Li, H. Du, G. Chen, S. H. Yeo, Viscoelastic properties of mr fluids under oscillatory shear, in: SPIE's 8th Annual International Symposium on Smart Structures and Materials, International Society for Optics and Photonics, 2001, pp. 333-342.

[21] J. A. Ruiz-López, J. C. Fernández-Toledano, D. J. Klingenberg, R. Hidalgo-Alvarez, J. de Vicente, Model magnetorheology: A direct comparative study between theories, particle-level simulations and experiments, in steady and dynamic oscillatory shear, Journal of Rheology 60 (1) (2016) 61-74.

[22] G. Bossis, E. Lemaire, Yield stresses in magnetic suspensions, Journal of Rheology (1978present) 35 (7) (1991) 1345-1354.

[23] T. McLeish, T. Jordan, M. Shaw, Viscoelastic response of electrorheological fluids. i. frequency dependence, Journal of Rheology (1978-present) 35 (3) (1991) 427-448.

[24] B. De Gans, C. Blom, A. Philipse, J. Mellema, Linear viscoelasticity of an inverse ferrofluid, Physical Review E 60 (4) (1999) 4518.

[25] J. A. Ruiz-López, J. C. Fernández-Toledano, R. Hidalgo-Alvarez, J. de Vicente, Testing the mean magnetization approximation, dimensionless and scaling numbers in magnetorheology, Soft matterdoi:10.1039/c5sm02267c.

[26] S. Melle, O. G. Calderón, M. A. Rubio, G. G. Fuller, Microstructure evolution in magnetorheological suspensions governed by mason number, Physical Review E 68 (4) (2003) 041503. 
[27] I. Petousis, E. Homburg, R. Derks, A. Dietzel, Transient behaviour of magnetic micro-bead chains rotating in a fluid by external fields, Lab on a Chip 7 (12) (2007) 1746-1751.

[28] M. T. López-López, P. Kuzhir, J. D. Durán, G. Bossis, Normal stresses in a shear flow of magnetorheological suspensions: viscoelastic versus maxwell stresses, Journal of Rheology (1978present) 54 (5) (2010) 1119-1136.

[29] J. Rodríguez-López, P. C. Blázquez, L. Elvira, F. M. de Espinosa, J. Ramírez, J. de Vicente, On the yielding behaviour in magnetorheology using ultrasounds, shear and normal stresses, and optical microscopy, Journal of Physics D: Applied Physics 48 (46) (2015) 465503.

[30] L. J. Felicia, J. Philip, Effect of hydrophilic silica nanoparticles on the magnetorheological properties of ferrofluids: A study using opto-magnetorheometer, Langmuir 31 (11) (2015) $3343-3353$.

[31] T. G. Kang, M. A. Hulsen, J. M. den Toonder, Dynamics of magnetic chains in a shear flow under the influence of a uniform magnetic field, Physics of Fluids (1994-present) 24 (4) (2012) 042001.

[32] B. De Gans, N. Duin, D. Van den Ende, J. Mellema, The influence of particle size on the magnetorheological properties of an inverse ferrofluid, The Journal of Chemical Physics 113 (5) (2000) 2032-2042.

[33] J. Ramos, D. Klingenberg, R. Hidalgo-Alvarez, J. de Vicente, Steady shear magnetorheology of inverse ferrofluids, Journal of Rheology (1978-present) 55 (1) (2011) 127-152.

[34] H. Ly, F. Reitich, M. Jolly, H. Banks, K. Ito, Simulations of particle dynamics in magnetorheological fluids, Journal of Computational Physics 155 (1) (1999) 160-177.

[35] E. Climent, M. R. Maxey, G. E. Karniadakis, Dynamics of self-assembled chaining in magnetorheological fluids, Langmuir 20 (2) (2004) 507-513. 
[36] R. Haghgooie, P. S. Doyle, Structure and dynamics of repulsive magnetorheological colloids in two-dimensional channels, Physical Review E 72 (1) (2005) 011405.

[37] M. R. Hashemi, M. T. Manzari, R. Fatehi, A SPH solver for simulating paramagnetic solid fluid interaction in the presence of an external magnetic field, Applied Mathematical Modelling $40(7-8)(2016) 43414369$.

[38] J. Dunwoody, The effects of inertia and finite amplitude on oscillatory plane shear flow of k-bkz fluids such as ldpe melts, Journal of non-Newtonian fluid mechanics 65 (2) (1996) 195-220.

[39] J. A. Yosick, J. A. Giacomin, W. E. Stewart, F. Ding, Fluid inertia in large amplitude oscillatory shear, Rheologica acta 37 (4) (1998) 365-373.

[40] R. H. Ewoldt, Nonlinear viscoelastic materials: bioinspired applications and new characterization measures, Ph.D. thesis, Massachusetts Institute of Technology (2009).

[41] J. L. Schrag, Deviation of velocity gradient profiles from the gap loading and surface loading limits in dynamic simple shear experiments, Transactions of The Society of Rheology (19571977) 21 (3) (1977) 399-413.

[42] J. Läuger, H. Stettin, Effects of instrument and fluid inertia in oscillatory shear in rotational rheometers, Journal of Rheology (1978-present) 60 (3) (2016) 393-406.

[43] R. Verberg, D. L. Koch, Rheology of particle suspensions with low to moderate fluid inertia at finite particle inertia, Physics of Fluids (1994-present) 18 (8) (2006) 083303.

[44] Y. K. Suh, S. Kang, Motion of paramagnetic particles in a viscous fluid under a uniform magnetic field: benchmark solutions, Journal of Engineering Mathematics 69 (1) (2011) 2558.

[45] M. R. Hashemi, M. T. Manzari, R. Fatehi, Evaluation of a pressure splitting formulation 
for weakly compressible SPH: Fluid flow around periodic array of cylinders, Computers \& Mathematics with Applications 71 (3) (2016) 758778.

[46] M. Shadloo, G. Oger, D. Le Touzé, Smoothed particle hydrodynamics method for fluid flows, towards industrial applications: Motivations, current state, and challenges, Computers \& Fluids $136(2016)$ 11-34.

[47] J. A. Stratton, Electromagnetic theory, John Wiley \& Sons, 1941.

[48] J. D. Jackson, Classical electrodynamics, Wiley, New York, 1962.

[49] D. C. Jiles, Introduction to magnetism and magnetic materials, CRC Press, 1998.

[50] D. J. Klingenberg, J. C. Ulicny, M. A. Golden, Mason numbers for magnetorheology, Journal of Rheology (1978-present) 51 (5) (2007) 883-893.

[51] J. de Vicente, D. J. Klingenberg, R. Hidalgo-Alvarez, Magnetorheological fluids: a review, Soft Matter 7 (8) (2011) 3701-3710.

[52] J. D. Ferry, Viscoelastic properties of polymers, John Wiley \& Sons, Inc, 1980.

[53] F. Ding, A. J. Giacomin, R. B. Bird, C.-B. Kweon, Viscous dissipation with fluid inertia in oscillatory shear flow, Journal of non-newtonian fluid mechanics 86 (3) (1999) 359-374.

[54] W. H. Li, H. Du, G. Chen, S. H. Yeo, N. Guo, Nonlinear viscoelastic properties of MR fluids under large-amplitude-oscillatory-shear, Rheologica acta 42 (3) (2003) 280-286.

[55] R. B. Bird, R. C. Armstrong, O. Hassager, C. F. Curtiss, Dynamics of polymeric liquids, Vol. 1, Wiley New York, 1977.

[56] G. Böhme, M. Stenger, On the influence of fluid inertia in oscillatory rheometry, Journal of Rheology (1978-present) 34 (3) (1990) 415-424. 


\section{Appendix A. Analytical Solution for Pure Newtonian Fluid under Oscillatory Shear}

The very first assumption to obtain an analytical solution for problem depicted in Fig. 4, is to consider a parallel fluid flow. Therefore, velocity vector has a non-zero component in the x-direction, $u=u(y, t)$, while other components are zero. The Navier-Stokes equation is reduced to

$$
\rho \frac{\partial u}{\partial t}=\eta_{0} \frac{\partial^{2} u}{\partial y^{2}}
$$

Considering the oscillatory boundary conditions for $u$,

$$
\begin{cases}u=-U_{0} \exp (\mathrm{i} \omega t) & y=0 \\ u=U_{0} \exp (\mathrm{i} \omega t) & y=h,\end{cases}
$$

the velocity field can be assumed as

$$
u=f(y) \exp (\mathrm{i} \omega t)
$$

Substituting Eq. (A.3) into Eq. (A.1) and solving the resulting differential equation for $f(y)$, one obtains

$$
f(y)=C_{1} \sin (\alpha y)+C_{2} \cos (\alpha y)
$$

where $\alpha=\sqrt{-(\mathrm{i} \rho \omega) / \eta_{0}}, C_{1}=U_{0}(\cos (\alpha h)+1) / \sin (\alpha h)$, and $C_{2}=-U_{0}$. Finally, Eq. (A.4) is substituted into Eq. (A.3), the real part is adopted and the apparent stress response is calculated as

$$
\tau_{x y}=\left.\eta_{0} \frac{\partial u}{\partial y}\right|_{y=0}
$$

